\title{
Evaluation of Difference of Urban and Rural Residents for the First Medical Consultation Site based on Propensity Score Match
}

\author{
Hongqing An, Yujia Kong, Wei Li ${ }^{*}$ \\ Weifang Medical University, China \\ *Corresponding author: Wei Li, Doctor, liwei@wfmc.edu.cn
}

\begin{abstract}
Objective: To study the difference of urban and rural residents for the first medical consultation Site and to expand the evaluating method for basic public health service accessibility. Methods: 1955 residents were randomly sampled from urban and rural district; Propensity score were used to balance the bias between groups; Statistical analysis was performed on the data to study the difference before and after the propensity score match. Results: After the propensity score match, age, culture degree, and insurance were balanced between groups. The difference of the first medical consultation site was not statistically significant between urban and rural Residents. Conclusion: After the basic public health service project, the first medical consultation site was not statistically significant from rural residents achieved the same; the effect of basic public health services in space is significant.
\end{abstract}

Keywords: propensity score match; the first medical consultation site; difference; urban; rural

\section{Introduction}

The report of the 18th Chinese Communist Party congress pledged to provide safe, effective, convenient and inexpensive public health service and basic medical service for Chinese people so as to improve their health level. To reach the goal of making basic medical and health services available to all citizens, we execute the important basic public health service project ${ }^{1}$. The evaluation of the implementation effect of the basic public health service project is the key point of the research for the people; the basic public health service accessibility is one of the most important indexes. Public health service accessibility is the degree of convenience the residents reach the primary health care institutions and spatial satisfaction they want for primary medical needs ${ }^{2}$. In this paper, we evaluate the difference of urban and rural residents for the first medical consultation Site, and analysis the method of the evaluation of the basic public health service accessibility.

\section{Data and variables}

\subsection{Data sources}

1955 residents over the age of 18 years were randomly sampled from urban and rural district. Myer index was 6.29 and sample is well.

The survey team consisted of the teachers and the graduate students with the knowledge of health management and statistics ask the question to the residents face to face and one by one.

\subsection{Study variables}

Status (rural=1, urban=2), Income (Yuan/year), Sex(male=1, female=2), Age (years), The first medical consultation Site (Village(community) clinics=1, Township hospital=2, country hospital=3, hospital at or above the country level=4), Education (under primary school=1, 
primary school $=2$, junior middle school $=3$, high middle school/technical school/secondary specialized school=4, college $=5$, university degree or more=6), Occupation (farmer and producer $=1$, operator $=2$, businessman $=3$, soldier $=4$, students $=5$, public employee $=6$, professionals=7, server=8, others=9), Nationality (Han=1, Hui=2, Uygur=3, Zhuang=4, others=5), The degree of difficulty to the living(without help $=1$, help from time to time $=2$, rely on the help=3), The distance to the basic medical and health institutions (meters), The distance to second medical and health institutions (li), Marriage(single $=1$, married $=2$, divorced $=3$, widowed $=4$ ), Medical insurance (without insurance $=0$, the new type of rural cooperative medical insurance $=1$, the basic medical insurance for working urban residents $=2$, the basic medical insurance for non-working urban residents $=3$, commercial insurance and others $=4$ ).

\subsection{Statistical analysis method}

We input the original data into the EpiDate3.1 database with the strictly controlled of quality so as to insure the integrity and accuracy of the data. We make propensity score matching by SAS9.2 and make statistical analysis by SPSS17.0 with the complete data. The caliper is $20 \%$ of two group propensity scores standard deviation.

The basic principle of the propensity score match is the influence factors of multiple covariates expressed by a propensity score ${ }^{3-4}$, then, balance the covariate distribution between different groups to control the bias with the propensity score match ${ }^{5}$.

In the application of propensity score match, it is important to the caliper value which is the key for the successful matching rate. If the caliper value is too large, the successful matching rate will be reduced. If the caliper value is too little, the evaluation results maybe incorrect though the successful matching rate increased for the cases of success matching reduced. In this paper, the caliper value is $20 \%$ of the propensity score standard deviations of two groups of urban and rural residents, it is the most suitable caliper value, it can reduce $98 \% \sim 99 \%$ of the bias ${ }^{6}$.

\section{Results}

\subsection{The basic situation}

Before the propensity score match, the total number of cases is 1955 . In these cases, rural and urban groups are 1252 cases and 703 cases. After the propensity score match, the total number of cases is 732 . In these cases, rural and urban groups are both 366 cases. The proportion of matching is $37.4 \%$.

\subsection{The covariate distributions before and after the propensity score match}

Before the propensity score match, age, culture degree, insurance are unbalanced $(\mathrm{P}<0.05)$, as show in table 1; after propensity score match, age, culture degree, insurance are balanced ( $\mathrm{P}>0.05)$, as show in table 2 .

\subsection{The first medical consultation site before and after the propensity score match}

Before the propensity score match, the difference of the first clinical institutions was statistically significant between urban and rural groups $(\mathrm{P}=0.035<0.05)$, as show in table 3 ; after propensity score matching, the difference of the first clinical institutions was not statistically significant between urban and rural groups $(\mathrm{P}=0.055>0.05)$, as show in table 4 . 
Table 1 - The covariate distributions before the propensity score match

\begin{tabular}{|c|c|c|c|c|c|}
\hline \multirow{2}{*}{ Covariates } & \multicolumn{2}{|c|}{ rural $(n=1252)$} & \multicolumn{2}{|c|}{$\operatorname{urban}(n=703)$} & \multirow{2}{*}{$P$} \\
\hline & number & percent & number & percent & \\
\hline Age & \multicolumn{2}{|c|}{$49.03 \pm 15.407$} & \multicolumn{2}{|c|}{$50.70 \pm 14.826$} & 0.020 \\
\hline Income & \multicolumn{2}{|c|}{$30771.88 \pm 31635.756$} & \multicolumn{2}{|c|}{$32233.00 \pm 40823.361$} & 0.379 \\
\hline Distance (meters) & \multicolumn{2}{|c|}{$358.97 \pm 450.475$} & \multicolumn{2}{|c|}{$332.18 \pm 293.660$} & 0.112 \\
\hline Distance (li) & \multicolumn{2}{|c|}{$11.73 \pm 11.540$} & \multicolumn{2}{|c|}{$10.94 \pm 12.608$} & 0.165 \\
\hline Education & & & & & 0.000 \\
\hline 1 & 171 & 13.7 & 174 & 24.8 & \\
\hline 2 & 345 & 27.6 & 177 & 25.2 & \\
\hline 3 & 368 & 29.4 & 225 & 32.0 & \\
\hline 4 & 150 & 12.0 & 67 & 9.5 & \\
\hline 5 & 66 & 5.3 & 5 & 0.7 & \\
\hline 6 & 152 & 12.1 & 55 & 7.8 & \\
\hline Sex & & & & & 0.076 \\
\hline 1 & 640 & 51.1 & 330 & 46.9 & \\
\hline 2 & 612 & 48.9 & 373 & 53.1 & \\
\hline Occupation & & & & & 0.438 \\
\hline 1 & 114 & 9.1 & 63 & 9.0 & \\
\hline 2 & 121 & 9.7 & 64 & 9.1 & \\
\hline 3 & 57 & 4.6 & 21 & 3.0 & \\
\hline 4 & 31 & 2.5 & 14 & 2.0 & \\
\hline 5 & 72 & 5.8 & 45 & 6.4 & \\
\hline 6 & 24 & 1.9 & 9 & 1.3 & \\
\hline 7 & 41 & 3.3 & 19 & 2.7 & \\
\hline 8 & 668 & 53.4 & 408 & 58.0 & \\
\hline 9 & 124 & 9.9 & 60 & 8.5 & \\
\hline Nationality & & & & & 0.082 \\
\hline 1 & 1202 & 96.0 & 683 & 97.2 & \\
\hline 2 & 16 & 1.3 & 1 & 0.1 & \\
\hline 3 & 20 & 1.6 & 14 & 2.0 & \\
\hline 4 & 5 & 0.4 & 1 & 0.1 & \\
\hline 5 & 9 & 0.7 & 4 & 0.6 & \\
\hline $\begin{array}{l}\text { The degree of } \\
\text { difficulty to the living }\end{array}$ & & & & & 0.912 \\
\hline 1 & 1129 & 90.2 & 635 & 90.3 & \\
\hline 2 & 85 & 6.8 & 45 & 6.4 & \\
\hline 3 & 38 & 3.0 & 23 & 3.3 & \\
\hline Marriage & & & & & 0.120 \\
\hline 1 & 169 & 13.5 & 69 & 9.8 & \\
\hline 2 & 971 & 77.6 & 565 & 80.4 & \\
\hline 3 & 28 & 2.2 & 17 & 2.4 & \\
\hline 4 & 84 & 6.7 & 52 & 7.4 & \\
\hline Medical insurance & & & & & 0.000 \\
\hline 0 & 29 & 2.3 & 14 & 2.0 & \\
\hline 1 & 873 & 69.7 & 47 & 6.7 & \\
\hline 2 & 324 & 25.9 & 590 & 83.9 & \\
\hline 3 & 9 & 0.7 & 16 & 2.3 & \\
\hline 4 & 17 & 1.4 & 36 & 5.1 & \\
\hline
\end{tabular}

Note: Qualitative variables test with $\chi^{2}$-test; quantitative variables test with t-test. 
Table 2 - The covariate distributions after the propensity score match

\begin{tabular}{|c|c|c|c|c|c|}
\hline \multirow{2}{*}{ Covariates } & \multicolumn{2}{|c|}{ rural $(n=366)$} & \multicolumn{2}{|c|}{ urban $(n=366)$} & \multirow{2}{*}{$P$} \\
\hline & number & percent & number & percent & \\
\hline Age & \multicolumn{2}{|c|}{$50.52 \pm 14.596$} & \multicolumn{2}{|c|}{$49.96 \pm 14.585$} & 0.602 \\
\hline Income & \multicolumn{2}{|c|}{$35393.17 \pm 46026.307$} & \multicolumn{2}{|c|}{$32483.61 \pm 44812.952$} & 0.386 \\
\hline Distance (meters) & \multicolumn{2}{|c|}{$378.60 \pm 468.105$} & \multicolumn{2}{|c|}{$323.01 \pm 285.215$} & 0.053 \\
\hline Distance (li) & \multicolumn{2}{|c|}{$11.23 \pm 12.828$} & \multirow{2}{*}{\multicolumn{2}{|c|}{$10.75 \pm 13.511$}} & 0.626 \\
\hline Education & & & & & 0.961 \\
\hline 1 & 74 & 20.2 & 72 & 19.7 & \\
\hline 2 & 88 & 24.0 & 86 & 23.5 & \\
\hline 3 & 109 & 29.8 & 109 & 29.8 & \\
\hline 4 & 43 & 11.7 & 47 & 12.8 & \\
\hline 5 & 8 & 2.2 & 5 & 1.4 & \\
\hline 6 & 44 & 12.0 & 47 & 12.8 & \\
\hline Sex & & & & & 0.553 \\
\hline 1 & 162 & 44.3 & 170 & 46.4 & \\
\hline 2 & 204 & 55.7 & 196 & 53.6 & \\
\hline Occupation & & & & & 0.165 \\
\hline 1 & 28 & 7.7 & 41 & 11.2 & \\
\hline 2 & 48 & 13.1 & 33 & 9.0 & \\
\hline 3 & 15 & 4.1 & 13 & 3.6 & \\
\hline 4 & 8 & 2.2 & 4 & 1.1 & \\
\hline 5 & 14 & 3.8 & 22 & 6.0 & \\
\hline 6 & 6 & 1.6 & 7 & 1.9 & \\
\hline 7 & 11 & 3.0 & 9 & 2.5 & \\
\hline 8 & 195 & 53.3 & 209 & 57.1 & \\
\hline 9 & 41 & 11.2 & 28 & 7.7 & \\
\hline Nationality & & & & & 0.794 \\
\hline 1 & 356 & 97.3 & 353 & 96.4 & \\
\hline 2 & 0 & 0 & 1 & 0.3 & \\
\hline 3 & 7 & 1.9 & 7 & 1.9 & \\
\hline 4 & 1 & 0.3 & 1 & 0.3 & \\
\hline 5 & 2 & 0.5 & 4 & 1.1 & \\
\hline $\begin{array}{l}\text { The degree of } \\
\text { difficulty to the living }\end{array}$ & & & & & 0.847 \\
\hline 1 & 330 & 90.2 & 326 & 89.1 & \\
\hline 2 & 24 & 6.6 & 28 & 7.7 & \\
\hline 3 & 12 & 3.3 & 12 & 3.3 & \\
\hline Marriage & & & & & 0.303 \\
\hline 1 & 34 & 9.3 & 38 & 10.4 & \\
\hline 2 & 300 & 82.0 & 302 & 82.5 & \\
\hline 3 & 5 & 1.4 & 9 & 2.5 & \\
\hline 4 & 27 & 7.4 & 17 & 4.6 & \\
\hline Medical insurance & & & & & 0.994 \\
\hline 0 & 14 & 3.8 & 14 & 3.8 & \\
\hline 1 & 47 & 12.8 & 47 & 12.8 & \\
\hline 2 & 285 & 77.9 & 285 & 77.9 & \\
\hline 3 & 3 & 0.8 & 2 & 0.5 & \\
\hline 4 & 17 & 4.6 & 18 & 4.9 & \\
\hline
\end{tabular}

Note: Qualitative variables test with $\chi^{2}$-test; quantitative variables test with t-test. 
Table 3 - The first medical consultation site before the propensity score match

\begin{tabular}{cccccc}
\hline \multirow{2}{*}{$\begin{array}{c}\text { the first medical } \\
\text { consultation site }\end{array}$} & \multicolumn{2}{c}{ rural $(n=1252)$} & urban $(n=703)$ & \multirow{2}{*}{$P$} \\
\cline { 2 - 4 } & number & percent & number & percent & \\
\hline 1 & 1070 & 85.5 & 565 & 80.4 & \\
2 & 108 & 8.6 & 80 & 11.4 & \multirow{2}{*}{0.035} \\
3 & 55 & 4.4 & 43 & 6.1 & \\
4 & 19 & 1.5 & 15 & 2.1 & \\
\hline
\end{tabular}

Table 4 - The first medical consultation site after the propensity score match

\begin{tabular}{cccccc}
\hline \multirow{2}{*}{$\begin{array}{c}\text { the first medical } \\
\text { consultation site }\end{array}$} & \multicolumn{2}{c}{ rural $(n=366)$} & \multicolumn{2}{c}{ urban $(n=366)$} & \multirow{2}{*}{$P$} \\
\cline { 2 - 4 } & numbe & percent & number & percent & \\
\hline 1 & 269 & 73.5 & 297 & 81.1 & \\
2 & 62 & 16.9 & 43 & 11.7 & \multirow{2}{*}{0.055} \\
3 & 25 & 6.8 & 22 & 6.0 & \\
4 & 10 & 2.7 & 4 & 1.1 & \\
\hline
\end{tabular}

3.4 The reason of the wish to the first medical consultation site after the propensity score match After the propensity score match, the difference of the reason of the wish to the first medical consultation site was not statistically significant between urban and rural groups $(\mathrm{P}=0.109>0.05)$, as show in table 5 :

Table 5 - The reason of the wish to the first medical consultation site after the propensity score match

\begin{tabular}{lcccc}
\hline \multirow{2}{*}{ Reason } & \multicolumn{2}{c}{ rural $(n=366)$} & urban $(n=366)$ \\
\cline { 2 - 5 } & number & percent & number & percent \\
\hline Convenient transportation & 87 & 23.8 & 102 & 27.9 \\
Shorter impatiens' waiting time & 95 & 26.0 & 95 & 26.0 \\
Excellent impatiens' equipment & 9 & 2.5 & 1 & 0.3 \\
Good technology & 23 & 6.3 & 19 & 5.2 \\
Good attitude towards patients & 40 & 10.9 & 56 & 15.3 \\
Reasonable price & 52 & 14.2 & 47 & 12.8 \\
Trust the doctor & 6 & 1.6 & 6 & 1.6 \\
Introduce by friends & 2 & 0.5 & 1 & 0.3 \\
Fixed medical institution & 52 & 14.2 & 39 & 10.7 \\
\hline
\end{tabular}

\section{Conclusion}

In this paper, we compared the difference of urban and rural residents for the first medical consultation site after the implementation of basic public health service in a certain area so as to evaluate the effect of the basic public health service accessibility. In general, the excellent evaluation method is the randomized controlled trial (RCT) ${ }^{7}$; it was called the gold standard. However, the survey data are not randomized strictly due to some objective or subjective factors ${ }^{8-9}$. There are some confounding factors leads to the inaccurate evaluation result of basic public health services ${ }^{10}$. Therefore, it is important to control the bias between groups effectively. The propensity score match is one of the excellent methods of balance the bias; it is an effective method for treatment the non-randomized data.

The research results show that the first medical consultation site is Village (community) clinics, the percent is 90 above. The difference of the first clinical institution of rural and urban residents was not statistically significant. The effect of basic public health services in 
time is significant. However, we should be reducing the gap of income and medical insurance between rural and urban residents ${ }^{11-12}$. Only in this way, we will achieve the goal of "everyone will have access to basic medical and health services in 2020" truly and completely.

On the other hand, the convenient transportation, the shorter impatiens' waiting time, increase the support of medical insurance are the main factors of the choice to the first clinics. The rural and urban residents' convenience of time and space will increase and the economic burden will reduce alone with continuous of the basic public health services. The difference of public health services accessibility between rural and urban residents.

\section{Acknowledgements}

The research work was supported by National Natural Science Foundation of China under Grant No. 71373181 and Natural Science Foundation of Shandong Provincial under Grant No. ZR2013HM045 and the Humanities and Social Science Project of Shandong Province under Grant No. J15WB43.

\section{References}

1. Y. Wei, Fairness Evaluation Analysis on Public Health Resource Allocation in China: Empirical Analysis Based on the Benchmarks of Fairness Analysis, Chinese health economics, 33(1), 2014, 32-34.

2. J. Huang, K. Li, M. Chen, Research on the theory and practice of the health service accessibility, Journal of Public Health and Preventive Medicine, 23(5), 2012, 60-62.

3. S. Wang, W. Meng, X. Wang, et al., Survival Analysis of Hepatocellular carcinoma patients based on propensity score matching, Chinese Journal of Health Statistics, 29(5), 2012, 680-682.

4. J. $L v, S$. Wang, The application of propensity score matching in evaluating chemotherapeutic effect of lung carcinoma, Chinese Journal of Health Statistics, 31(2), 2014, 190-192.

5. M. Wu, C. Wu, R. Wang, et al., Estimation and Comparison of Methods of value propensity score method, Chinese Journal of Health Statistics, 30 (3), 2013, 440-444.

6. X. Zhao, B. Li, S. Wang, et al., Effect Evaluation between GP and NP Regiments in Treatment of Locally Advanced Non-small Cell Lung Cancer Based on Propensity Score Matching, Chinese Journal of Health Statistics, 31(1), 2014, 34-36.

7. S. Hong, Y.N. Youn, G. Yi, et al., Long Term Results of ST-Segment Elevation Myocardial Infarction versus Non-ST-Segment Elevation Myocardial Infarction after Off-Pump Coronary Artery Bypass Grafting: Propensity Score Matching Analysis, J Korean Med Sci, 27(2), 2012, 153-159.

8. K.H.Oh, Y.H. Hwang, J.H. Cho, et al., Outcome of early initiation of peritoneal dialysis in patients with end-stage renal failure, J Korean Med Sci, 27(2), 2012, 170-176.

9. J.P. Wisnivesky, E.A. Halm, M. Bonomi, et al., Postoperative radiotherapy for elderly patients with stage III lung cancer, Cancer, 2(13), 2012, 265-285.

10. G. Apolone, S. Deandrea, M. Montanari, et al., Evaluation of the comparative analgesic effectiveness of transdermal and oral opioids in cancer patients: A propensity 
score analysis, Eur J Pain, 16(2), 2012, 229-238.

11. X.Wang, The analysis of dynamic relationship about social basic medical insurance and health cost, Chinese Journal of Health Statistics, 31(1), 2013, 77-80.

12. H.Wu, G. Sun, X. Chen, et al., Comprehensive assessment on implementation of national basic public health services package, Chinese Journal of Public Health, 30(6), 2014, 715-717. 